\title{
Response-Based Strengthening in Task Shifting: Evidence From Shift Effects Produced by Errors
}

\author{
Marco Steinhauser and Ronald Hübner \\ Universität Konstanz
}

\begin{abstract}
The hypothesis is introduced that 1 source of shift costs is the strengthening of task-related associations occurring whenever an overt response is produced. The authors tested this account by examining shift effects following errors and error compensation processes. The authors predicted that following a specific type of error, called task confusion, shift benefits instead of shift costs should result. A series of 3 experiments confirmed this prediction showing that task confusions produce shift benefits in subsequent trials (Experiment 1), even when the error is detected (Experiment 2). Moreover, only posterror processes that imply an error correction response produce shift costs (Experiment 3 ). These results additionally suggest that error detection cannot prevent errors from affecting subsequent performance.
\end{abstract}

Keywords: task shifting, error processing, response repetition

The task-shifting paradigm is currently a widely applied method for investigating mental control processes involved in the configuration, coordination, and execution of simple tasks. In a typical experiment, the participants have to shift between two tasks in random or alternating order. This usually results in so-called shift costs, that is, increased response times and error rates on task-shift trials relative to task-repetition trials (Allport, Styles, \& Hsieh, 1994; Meiran, 1996; Rogers \& Monsell, 1995). Originally, it was supposed that shift costs directly indicate mental control processes. Meanwhile, however, many results suggest that they also reflect proactive effects resulting from the performance on preceding trials. As a consequence, some authors hypothesized that at least a portion of the shift costs are the result of previous activation and inhibition processes (e.g., Allport et al., 1994; Schuch \& Koch, 2003), whereas others proposed that learning or binding processes are involved (e.g., Meiran, 2000a; Wylie \& Allport, 2000).

Up to now it is open for discussion which theory accounts best for the shift costs, or whether both mechanisms contribute. Therefore, the aim of the present study was to provide additional evidence for answering this question. For attaining this objective, we examined effects that are usually ignored in task-shift experiments, namely, shift costs produced by errors. Normally, posterror trials are discarded from the analyses of shift costs. However, shift costs caused by errors are of great interest. Their analysis can be used not only to test theories of task-shift costs, but also to improve our general understanding of how errors affect behavior.

We will develop our approach by first considering the proposed theories of shift costs in some detail. Then, a short overview of

Marco Steinhauser and Ronald Hübner, Fachbereich Psychologie, Universität Konstanz.

This work was supported by Grant DFG: Hu 432/8-1 to Ronald Hübner from the Deutsche Forschungsgemeinschaft. We would like to thank Michel D. Druey for helpful comments.

Correspondence concerning this article should be addressed to Marco Steinhauser, Universität Konstanz, Fachbereich Psychologie, Fach D29, D-78457, Konstanz, Germany. E-mail: Marco.Steinhauser@uni-konstanz.de theories on error processing and error compensation will be given. Finally, after we have combined these areas to derive our hypotheses, a series of three experiments are reported in which these ideas were tested.

\section{Shift Costs: Carryover of Task Activation or Associative Learning?}

It is widely assumed that mainly two components contribute to shift costs (Meiran, 1996; Rogers \& Monsell, 1995; Ruthruff, Remington, \& Johnston, 2001; Sohn \& Anderson, 2001). One component is due to endogenous reconfiguration processes such as goal implementation. However, this component contributes to the costs only if the tasks cannot fully be prepared in advance. In contrast, the other component can be observed even with a sufficiently long preparation period. Although most researchers agree that these so-called residual costs are due to proactive effects, the specific mechanisms are still under dispute. Mainly two classes of models have been considered in this respect: activation-based accounts and learning accounts.

The activation-based accounts assume that during task performance the relevant and irrelevant tasks are activated and inhibited, respectively. Relative activation and inhibition are then propagated to the next trial where they affect performance (Allport et al., 1994). In particular, performing a previously activated task is beneficial, whereas performing a previously inhibited task produces costs. This account is supported, for instance, by experiments in which the shift cost on a given trial increased with the degree of task inhibition on the previous trial (Allport et al., 1994; Goschke, 2000; Hübner, Kluwe, Luna-Rodriguez, \& Peters, 2004; Mayr \& Keele, 2000). Within such a framework, the shift costs reflect an aftereffect of mechanisms involved in the selection of the previous response (Schuch \& Koch, 2003). The inhibition and activation of tasks are required to guarantee reliable responding, whereas their effects on subsequent performance are mere by-products.

In contrast, the learning accounts assume that the proactive effects reflect learning processes, which take place during task 
execution to affect subsequent behavior. The involvement of memory processes in task shifting has initially been suggested to account for item-specific effects on the shift cost. For instance, Wylie and Allport (2000) proposed that task execution strengthens the associations between stimulus attributes (e.g., the color red in a colored word) and their responses. These associations are automatically retrieved when the same stimulus is presented on later trials and, in this way, affect performance. One result that supports such an account is that stimuli that have been performed more frequently with the alternative task produce stronger shift costs (Waszak, Hommel, \& Allport, 2003; Wylie \& Allport, 2000).

Learning accounts, however, could also account for the shift costs themselves. In the model of Meiran (2000a), task execution leads to a binding between the task-specific stimulus categories and the respective response set (what Meiran terms the adjustment of the response task set). Assume, for instance, that the judgments odd/even and less/greater than 5 are mapped on the same left and right responses. In this case, performing the odd/even judgment leads to a binding of the response categories left and right to the stimulus categories odd and even, respectively. A subsequent shift to the alternative judgment is impaired because the response categories are more strongly bound to the now irrelevant stimulus categories. Although both responses are bound to their categories on each trial, the binding of the actually produced response was assumed to be slightly stronger, which should account for the pattern of response repetition effects typically observed in task shifting. In contrast to the mere activation-based accounts, this model suggests a mechanism that modifies the respective associations after task execution has finished. Meiran (2000a) assumed that this binding is not transient but affects the immediately following trial as well as later performance. Therefore, it can be viewed as a learning process. ${ }^{1}$

Some authors even suggested a combination of activation-based and learning accounts. Waszak et al. (2003), for instance, proposed that item-specific effects reflect the associative strength of single stimulus-task associations, whereas the shift costs result from the increased activation of all associations of a single task (see also Gilbert \& Shallice, 2002). However, one could also hypothesize that both effects result from the same learning mechanisms. For instance, whereas item-specific effects are due to the associative strength of stimulus-task associations, the shift costs reflect the learning or binding of task-response associations in the sense of Meiran (2000a). Furthermore, if we assume that the degree of associative strengthening is not constant but depends on the current activation of the task sets, this could additionally explain why the shift costs are related to the amount of activation and inhibition on the preceding trial. In other words, task-set activation might affect task performance on the subsequent trial only indirectly by modulating the amount of strengthening.

If we assume that such a combined account is valid, a crucial question is at which stage the strengthening takes place. A plausible candidate is response production. At this stage, the chosen response as well as the task that determined the response are maximally active. Therefore, strengthening would be most effective at this time. However, it is not necessary to assume that the response directly triggers strengthening. Instead, it is possible that the mental system merely uses response production as a cue for initiating a learning process. Accordingly, we call this account the response-based strengthening hypothesis. The term response- based should express that producing an overt response is crucial for learning to occur. This hypothesis is supported by results from Schuch and Koch (2003), who found no shift costs after no-go trials and, therefore, concluded that the inhibition of the irrelevant task during response selection is crucial for shift costs (but see also Kleinsorge \& Gajewski, 2004). However, these results could also be interpreted in the sense that, since no specific response was initiated on no-go trials, no strengthening occurred.

In the experiments reported here, the response-based strengthening hypothesis was tested against the pure activation-based account. We did not address the question of what exactly is strengthened to produce the shift costs. Rather, we tested the crucial prediction of the response-based strengthening account that task execution affects subsequent performance only if an overt response is produced. We will show that activating a task does not affect subsequent performance if this response is not produced overtly. To achieve this, we compared conditions in which the task that was activated before response production differed from the task that was activated after response production. The crucial question then was which of the two tasks determined the shift costs on the subsequent trial. Fortunately, such a situation is given when an error occurred under certain conditions. To see how this can be, we have first to consider some general results and theories from research on error processing.

\section{Error Processing and Error Compensation}

If one considers the literature on error processing, then one learns that errors initiate at least two processes: immediate error processing and strategy changes. Immediate error processing has intensively been examined by Rabbitt and his colleagues, who focused mainly on the mechanisms of error detection (for a review, see Rabbitt, 2002). They employed two types of error-detection responses. Their participants either had to correct errors immediately by pressing the correct response key (error-correction response, ECR), or they had to indicate a detected error by pressing a neutral response key (error-signaling response, ESR). The durations of error signaling and error correction were measured by the time elapsed between the erroneous response and the respective detection response. Rabbitt found that ECRs occurred faster and more frequently than ESRs and concluded that the former responses are more automatic than the latter (Rabbitt, 1990, 2002). This was further supported by the fact that fast and spontaneous error corrections sometimes occurred even though they were not required.

An interesting question is how errors are detected. Depending on the specific type of error, several hypotheses have been proposed. For instance, motor errors, which result from a correct response selection combined with a wrong response production, can simply be detected by comparing the actual response with the selected one (Megaw, 1972; Scheffers \& Coles, 2000). The situation is more complex, however, for perceptual and response

\footnotetext{
${ }^{1}$ Although Meiran (2000a) used the binding terminology within his model and explicitly referred to a learning process in Meiran and Gotler (2001), he mentioned that the binding of stimulus categories to responses can also be understood as activating category-response rules (Meiran, 2000a). Therefore, the model can be viewed as a hybrid model combining activation-based as well as learning and binding processes.
} 
selection errors. In these cases, one has also to explain how the system knows what the correct response should have been. Several models have been discussed (see Rabbitt, 1967), all of which share the idea of the extended processing hypothesis (Rabbitt \& Vyas, 1981). According to this hypothesis, evidence accumulation continues after a response and an error is detected if this processing yields a result that differs from the given response (for a comparable view, see Yeung, Cohen, \& Botvinick, 2004). Evidence for the extended processing hypothesis has been provided by a number of studies (e.g., Rabbitt \& Rodgers, 1977; Rabbitt \& Vyas, 1981).

In addition to eliciting immediate processes, errors can also influence response strategies. The most established phenomenon is the so-called posterror slowing (Fairweather, 1978; Laming, 1979a, 1979b), that is, the increase in response time after an error. It is assumed that this results from an adaptation of the speedaccuracy trade-off in the direction of a lower error probability. Further evidence for a strategy change was provided by Ridderinkhof (2002). By applying a stimulus-response compatibility task, he found that automatic response activation by an irrelevant stimulus dimension was inhibited more strongly after an error.

\section{Errors in Task-Shift Experiments}

After these short reviews on task-shift mechanisms and error related processes, we can consider how these areas fit together, that is, how errors arise in task-shift experiments and which processes they initiate. First of all, compared to more simple paradigms, task-shift experiments have an additional source of errors. Since each task can usually be applied to each stimulus, a presented stimulus activates both tasks in a bottom-up manner (Rogers \& Monsell, 1995; Steinhauser \& Hübner, in press-a; Waszak et al., 2003). This leads to a task conflict, which is presumably resolved by suppressing the irrelevant task (Mayr, 2001; Steinhauser \& Hübner, in press-b). However, on some trials suppression fails so that the irrelevant task determines the response and causes an error.

Thus, we can distinguish mainly two error sources in task-shift studies. First, there are response confusions that occur for several reasons (e.g., noise in the response selection stage) even though the correct task was applied. ${ }^{2}$ Additionally, there are task confusions that result from the correct application of the wrong task. It is important to note that task confusions do not necessarily produce an error response. For congruent stimuli, that is, stimuli that require the same response for each task, the correct application of the wrong task also produces a correct response. For the objective of the present study, task confusions are of foremost interest, because only errors resulting from this source should systematically affect shift costs.

The interesting question now is how errors caused by task confusions affect shift costs. Obviously, the answer depends on the specific account of the shift costs. A rather simple prediction results from a response-based strengthening account. If Task B determines the response although Task $\mathrm{A}$ was intended, then associations between Task B and the responses are strengthened. This follows because, at the stage where the response is initiated and strengthening occurs, the wrong task (B) is more activated than the correct task (A). Accordingly, on the next trial Task B should be easier than Task A. As a consequence, after a task confusion, shift benefits ${ }^{3}$ should be observed instead of shift costs, at least as long as the error is not corrected.

On the other hand, the activation-based account predicts that the final state of the task activations on a given trial affects the performance on the subsequent trial. Thus, if we assume that the state of task activations, which determined the current response, propagates to the next trial, and that this also holds for error trials, then the same prediction can be derived as for the response-based strengthening account. However, as we have seen, there are good reasons to question that the state of activations that determined the response remains unaltered on error trials. According to the extended processing hypothesis (Rabbitt \& Vyas, 1981), evidence accumulation continues until the correct response is available. Consequently, the activation of the correct task increases during the extended processing while that of the wrong task decreases. As a result, at the end of a trial with a detected error the relative activation of both tasks is similar to that after a trial with a correct response. Thus, if we take the extended processing hypothesis into account, then the activation-based account predicts that detected errors should produce shift costs.

\section{The Present Paradigm}

The considerations so far show that error processing models are helpful in deriving specific predictions for the different shift-cost accounts. The following series of task-shift experiments was designed to test these predictions. In these experiments, parity and magnitude judgments varied randomly across trials. On each trial, a cue indicated the required judgment, and a relatively long cuestimulus interval allowed plenty of time for preparation. Furthermore, since the error rates are usually rather low $(<10 \%)$ in task-shift experiments, a deadline procedure was used in order to obtain more errors. A deadline normally eliminates variance from the response times. Therefore, we expected the effects to be more pronounced in the error rates than in the response times. Because of this, we focused on the shift costs as measured by the difference in error rate between task repetitions and task shifts.

In the first experiment, we examined whether errors due to task confusions produce shift costs or shift benefits. The interpretation of the data relies on additional assumptions that were tested in the subsequent two experiments.

\section{Experiment 1}

The aim of the first experiment was to examine whether errors caused by task confusion produce shift costs or shift benefits. However, how can we know whether an error resulted from task confusion or from response confusion? Fortunately, there is an indirect way to separate these error types. We made use of the fact that the two error types have different consequences for congruent

\footnotetext{
${ }^{2}$ We used the formulation applying a task for simplicity. It implies only that this task had the highest activation and, accordingly, contributed most strongly to the selection of a response.

${ }^{3}$ When using the terms shift costs and shift benefits, we implicitly use a definition of task repetitions and task shifts that refers to the tasks indicated by the cues. If Task $\mathrm{A}$ is required on trial $N$ and Task $\mathrm{B}$ is required on trial $N-1$, trial $N$ is viewed as a task-shift trial, irrespective of whether the participant erroneously applied Task A on trial $\mathrm{N}-1$ or not.
} 
and incongruent stimuli, that is, for stimuli that require the same or different responses for each task, respectively.

For congruent stimuli, errors can be due only to response confusions. This follows from the fact that task confusions do not lead to an error because the irrelevant task requires the same response as the relevant one. Consequently, the error rate and the rate of correct responses contain the same proportion of task confusions. This implies that error responses should lead to the same shift effects as correct responses, which are a mixture of the effects produced by correct task applications and task confusions.

In contrast, for incongruent stimuli each task confusion produces an error (with the exception of trials where both a task confusion and a response confusion occurs) because the irrelevant task now requires a different response as the relevant one. Therefore, the error rate should contain a larger proportion of task confusions than the rate of correct responses. One could even expect that most of the errors on incongruent stimuli are due to task confusions because response confusions should be rather rare on simple tasks like the present ones. Moreover, if task confusions produce a task-shift benefit instead of costs, as predicted by the response-based strengthening hypothesis, the shift costs should be reversed after an error for this stimulus type.

Taken together, our hypothesis predicts that errors for congruent stimuli produce shift effects on subsequent trials that differ from those produced by errors for incongruent stimuli. Thus, our analysis focuses on testing a three-way interaction between the factors Task Transition (task repetition, task shift), Accuracy on trial $\mathrm{N}-1$ (correct, error), and Congruency on trial $\mathrm{N}-1$ (congruent, incongruent).

Response repetition effects which are also important for the interpretation of shift effects (e.g., Hübner \& Druey, in press; Meiran, 2000a; Rogers \& Monsell, 1995; Schuch \& Koch, 2004) were not considered in this and the following experiments. However, they are reported in a separate section at the end of the last experiment.

\section{Method}

Participants. Twelve participants ( 8 female, 4 male) between 19 and 39 years of age (mean 25.3) with normal or corrected-to-normal vision participated in the study. Participants were recruited at the University of Konstanz and were paid 5 euros per hour.

Apparatus. The stimuli were presented on a 21-inch color monitor. An IBM-compatible PC controlled stimulus presentation and response registration.

Stimuli. Stimuli were the digits 2 to 9 comprising a width of $1.24^{\circ}$ visual angle and a height of $1.77^{\circ}$ visual angle at a viewing distance of 127 $\mathrm{cm}$. A circle and a square, both $1.43^{\circ}$ in diameter, were used as cues. Cues and stimuli were presented in white color on a black background.

Design and procedure. On each trial, participants had to apply one of two judgments to a digit presented on the screen. In the parity judgment, the digit had to be classified as odd or even. In the magnitude judgment, it had to be classified as less than five or greater than five. Responses were given by pressing a response button with the index finger (even, less than 5 ) or the middle finger (odd, greater than 5) of the right hand.

Each trial started with the presentation of the cue for $300 \mathrm{~ms}$ followed by a blank screen for $900 \mathrm{~ms}$. The stimulus was presented for $150 \mathrm{~ms}$ followed by a blank screen. After a specific interval, an acoustical deadline signal $(800 \mathrm{~Hz})$ appeared for $150 \mathrm{~ms}$. Participants were instructed to respond faster than this signal. A new trial started $1000 \mathrm{~ms}$ after the response. No feedback on the accuracy of the response was provided. However, on some trials a speed feedback was given. Whenever the response time exceeded the deadline on five consecutive trials, the German word schneller [faster] was presented on the screen $200 \mathrm{~ms}$ after the response for $200 \mathrm{~ms}$.

Participants worked through 30 blocks of 64 trials, resulting in a total amount of 1920 trials. Within each block, the order of judgments was randomized. The stimuli never repeated because it is known that stimulus repetitions are special cases with respect to task-shifting effects (Hoffmann, Kiesel, \& Sebald, 2003; Hübner et al., 2004). Moreover, stimulus repetitions imply that congruency also repeated and therefore occur only in some cells of our design. The blocks were distributed on three experimental sessions. These sessions were preceded by a practice session. The practice session started with five blocks in which no deadline signal was provided, and participants were instructed to respond as fast and accurately as possible. These were followed by five blocks with the deadline signal. In these blocks, a speed feedback was given on each trial in which the deadline was exceeded.

The deadline, that is, the interval between the onset of the stimulus and the onset of the acoustical signal, was determined in two steps. During Practice Blocks 6-10, the deadline equaled the median response time of Practice Block 5, that is, the last block without a deadline. At the beginning of the first experimental session, a new deadline was determined by computing the median response time of Practice Block 10, that is, the last block in the practice session.

\section{Results}

Trials following a speed feedback $(0.9 \%)$ and trials following a spontaneous correction response $(1.8 \%)$ were excluded from further analyses. Error rates as well as mean response times of correct responses were analyzed by three-way ANOVAs with repeated measurement on the factors Task Transition (task repetition, task shift), Accuracy on trial $N-1$ (correct, error), and Congruency on trial $N-1$ (congruent, incongruent).

Error rates. In this and the following experiments, an arcsine transformation was applied for analyzing the frequency data (Winer, Brown, \& Michels, 1991). The mean error rate was $24.4 \%$. The error rates for congruent and incongruent stimuli were $21.8 \%$ and $26.9 \%$, respectively. For simplicity, we will report only effects involving the factor Accuracy on trial $N$-1. Planned comparisons on shift costs and shift benefits were conducted by using one-sided $t$ tests.

The main results are presented in Figure 1. The interaction between Accuracy on trial $\mathrm{N}-1$ and Task Transition was significant, $F(1,11)=7.52, p<.05$. However, this effect was qualified by a three-way interaction involving all factors, $F(1,11)=6.60$, $p<.05$. When the stimulus on trial $N-1$ was congruent, small but not significant shift effects were observed after a correct response [repetition: $23.6 \%$; shift: $24.6 \% ; t(11)=1.00 ; p=.17$ ] and after an error [repetition: $24.9 \%$; shift: $24.6 \% ; t(11)=0.17 ; p=.43$ ] However, if the stimulus on trial $N-1$ was incongruent, there were significant shift costs after a correct response [repetition: $23.0 \%$; shift: $26.4 \% ; t(11)=3.51 ; p<.01]$ but reliable shift benefits after an error [repetition: $26.0 \%$; shift: $21.8 \% ; t(11)=2.78 ; p<.01$ ]

Since this interaction is directly related to our hypotheses, we analyzed the data separately for trials following a congruent stimulus and those following an incongruent stimulus. On trials following a congruent stimulus, the interaction between the factors Accuracy on trial $\mathrm{N}-1$ and Task Transition was not significant, $F(1,11)=0.45, p=.52$. However, on trials following incongruent stimuli, both factors interacted significantly, $F(1,11)=15.7$, $p<.01$. 


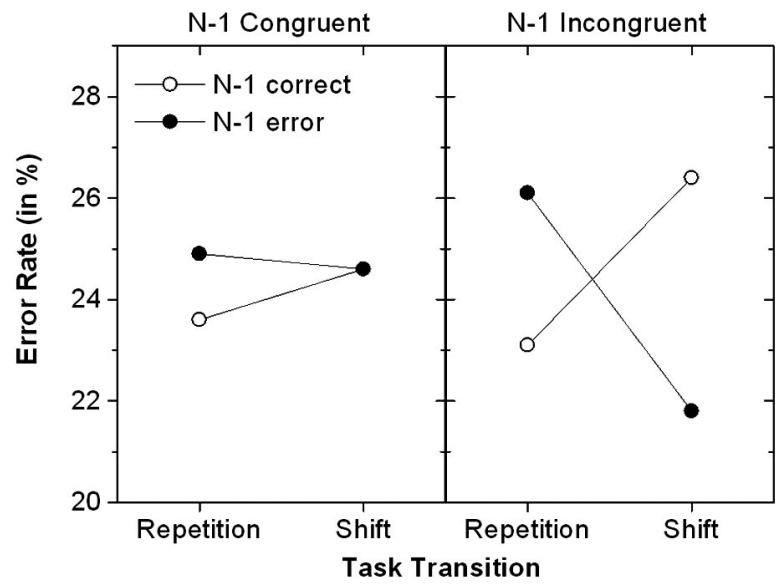

Figure 1. Error rates for task shifts and task repetitions following congruent and incongruent stimuli, and following correct responses and errors, from Experiment 1.

Response times. For the analysis of the response times, we additionally excluded trials with response times less than or greater than two standard deviations below or above the mean computed for each condition and each participant. Using this procedure, $2.5 \%$ of trials were removed. The overall mean was $324 \mathrm{~ms}$. No significant effects were obtained. However, a marginally significant interaction between Task Transition, Accuracy on trial $\mathrm{N}-1$, and Congruency on trial $\mathrm{N}-1$ indicated a trend in the same direction as in the error rates, $F(1,11)=3.53, p=.09$. On trials following incongruent stimuli, shift costs emerged after a correct response [repetition: $323 \mathrm{~ms}$; shift: $329 \mathrm{~ms} ; t(11)=2.26, p<.05$ ], while shift benefits were observed following an error [repetition: $330 \mathrm{~ms}$; shift: $322 \mathrm{~ms} ; t(11)=1.95, p<.05]$. On trials following a congruent stimulus, small but not significant shift costs were obtained following correct responses [repetition: $324 \mathrm{~ms}$; shift: $326 \mathrm{~ms} ; t(11)=0.70, p=.25$ ] as well as following errors [repetition: $314 \mathrm{~ms}$; shift: $320 \mathrm{~ms} ; t(11)=1.14, p=.14$ ]. It is interesting that the mean response time for responses after an error (321 ms) was similar to that for responses following a correct response $(326 \mathrm{~ms}), F(1,11)=0.96, p=.35$. In other words, there was no posterror slowing.

\section{Discussion}

In the present experiment, it was tested whether errors caused by task confusions produce shift costs or shift benefits on subsequent trials. Depending on the outcome, either the response-based strengthening model or the activation-based account is supported. First of all, the results show that correct responses produced the usual shift costs. However, as predicted by the response-based strengthening model, shift benefits occurred after an error on trials with an incongruent stimulus. Since errors on these trials can result from task confusion, we take this as evidence that the wrong task determined the response and, accordingly, that the wrong task became associated with the responses. Consequently, a shift to the erroneously applied task on the subsequent trial was beneficial, whereas a repetition of the previously intended task was costly.

As expected, substantial shift costs and benefits were observed only on trials following an incongruent stimulus. This resulted from the fact that task confusions produced an error only for incongruent stimuli. Thus, only on trials with incongruent stimuli, task confusions were more frequent on errors than on correct responses. As a consequence, errors produced different shift effects on subsequent trials than correct responses. On the other hand, for congruent stimuli, task confusions should have produced mostly a correct response. Therefore, the same number of task confusions should have occurred for erroneous and for correct responses. However, because task confusions led to shift benefits on the subsequent trial, on average they compensated the shift costs resulting from the application of the correct task to some degree. This is why the costs and benefits after a congruent stimulus were largely reduced. ${ }^{4}$

Although our predictions were mostly confirmed, one might wonder whether the similar error rates for congruent and incongruent stimuli $(21.8 \%$ vs. $26.9 \%)$ contradict the assumption that the majority of errors for incongruent stimuli were caused by task confusions. To examine whether this was indeed the case, we estimated the relative frequencies of task confusions and response confusions for both stimulus types by means of a simple multinomial model (see Appendix for details). For applying this model, certain assumptions had to be made that probably did not reflect the exact mechanisms underlying task performance. Nevertheless, the estimated frequencies can be seen as approximate figures.

The model reveals that for incongruent stimuli $26.3 \%$ of the errors and $2.7 \%$ of the correct responses are due to task confusions. On the other hand, for congruent stimuli $9.0 \%$ of both errors and correct responses are based on task confusions. These values nicely account for our pattern of shift effects on subsequent trials. For incongruent stimuli, task confusions were about 10 times as often responsible for errors as for correct responses. In contrast, for congruent stimuli the same portion of errors and correct responses was produced by task confusions.

One might ask whether it makes sense to assume that the $26.3 \%$ of the errors, which were due to task confusions on incongruent trials, led to a reversal of shift costs, when on the remaining $73.7 \%$ of the error trials the correct task was applied. Fortunately, there is a reasonable answer to this question. One simply has to assume that the shift effects resulting from errors due to a task confusion (together with a correct response) are stronger than the shift effects resulting from errors due to a response confusion (together with the correct task). In this case, the net result could be a shift benefit because the strong shift benefits from the task confusions could outweigh the small shift costs from the response confusions. The question remains why the shift effect produced by a response confusion should be smaller than that caused by a task confusion.

\footnotetext{
${ }^{4} \mathrm{~A}$ reduction of shift costs following congruent stimuli has also been observed in other studies (Goschke, 2000; Monsell et al., 2003). These authors explained this effect in another way. According to them, incongruent stimuli require additional inhibition of the alternative task, which is propagated to the next trial and leads to increased shift costs. We think that both mechanisms-fewer task confusions on correct responses of congruent stimuli as well as increased inhibition on correct responses of incongruent stimuli-could contribute to the effect. As mentioned earlier, more inhibition of the alternative task should increase the relative activation of the relevant task, which in turn implies more strengthening of this task.
} 
This can be explained by our earlier suggestion that strengthening occurs gradually. In particular, we suggested that the amount of strengthening (i.e., the change of the associative weights) is proportional to the state of activation of the two tasks. If the dominant task is only slightly more activated than the nondominant task, then only weak shift effects should be produced. In case the dominant task is strongly activated, strong shift effects should result. Now, it is reasonable to assume that the response selection for a weakly activated task is strongly affected by noise, which frequently leads to response confusions. Consequently, the shift effects produced by response confusions should be generally smaller. It is interesting that this reasoning also predicts that the shift costs produced by errors on congruent stimuli should be generally smaller than those produced by correct responses because, on congruent stimuli, errors are always due to response confusions (irrespective of whether an additional task confusion has occurred). Indeed, such a trend can be observed in our data, at least for the error rates (see Figure 1).

Taken together, the observed shift benefits indicate that the wrong task was able to determine the response and, as a consequence, caused a proactive effect on the following trial. According to the response-based strengthening account, the shift effects reflect the strengthening of associations between the task and the response at the time the response was produced. On the other hand, ctivation-based account assumes that the shift effects measured on a specific trial reflect the activation of the tasks at the beginning of this trial. Therefore, if posterror processes altered the relative activation of the tasks, they should have influenced the shift effects. Since error detection requires that the correct task becomes activated during posterror processing, we would have observed shift costs following an error. Obviously, this was not the case. Thus, the activation-based account can be rejected.

However, one might argue that no error detection in the sense of Rabbitt and Vyas (1981) took place during posterror processing or that this had no effect on subsequent performance. That is, it could be that there was no posterror phase when the intended task was activated. However, this implies that errors were not detected in our experiment. This is not implausible if we consider the fact that no posterror slowing was observed. Possibly, error detection is not an automatic process as suggested by Rabbitt (2002), but rather requires that participants intend to detect errors.

In order to determine which explanation is correct, one needs to know whether errors that caused shift benefits were detected. If this was the case, then it can be concluded that posterror processes occurred. In the following experiment, this question was examined by using a direct measure of error detection.

\section{Experiment 2}

In this experiment, the participants were to respond as fast as possible with an ESR (error-signaling response; Rabbitt, 1968) whenever they detected an error. ESRs were given by pressing the space bar of a keyboard with all fingers of the left hand, while the actual responses were given with the right hand. ESRs were required in half of the blocks, while no such responses had to be given in the other half. Furthermore, since the modulation of shift effects by a preceding error was observed only when the preceding stimulus was incongruent, we exclusively presented incongruent stimuli in this experiment.

In contrast to Experiment 1, participants were now encouraged to detect their errors. If error detection takes place, then posterror processes should occur that determine the correct response and, to achieve this, activate the correct task. Accordingly, if shift effects reflect the relative activation of the tasks, we would expect that detected errors produce shift costs and not shift benefits on the subsequent trial. If this prediction does not hold, the responsebased strengthening account is supported. This view assumes that it is not the activation of the tasks that predicts subsequent shift effects but rather the task that determined the erroneous response. That is, shift benefits would indicate that the wrong task determined the response even though the correct task was activated during posterror processing.

\section{Method}

Fourteen participants ( 9 female, 5 male) between 21 and 32 years of age (mean 23.7) with normal or corrected-to-normal vision participated in the study. Participants were recruited at the University of Konstanz and were paid 5 euros per hour.

Stimuli and tasks were the same as in Experiment 1. Here, however, only the incongruent digits $1,3,6$, and 8 were used. Furthermore, two block types were administered. In the ESR condition, participants were instructed to press the space bar of a keyboard with all fingers of the left hand as fast as possible whenever they had made an error. The ESR had to occur within $1000 \mathrm{~ms}$ after the response; otherwise, a new trial was started. If an ESR was given, the new trial started $1000 \mathrm{~ms}$ after the ESR. In the remaining blocks, no ESR was required. The two block types alternated, and the first block in a session was counterbalanced across participants.

Since only four stimuli were used, the predictability of the next stimulus was minimized by allowing stimulus repetitions. However, stimulusrepetition trials were excluded from our main analyses.

\section{Results}

\section{Errors and Error-Signaling Responses}

First of all, we analyzed the relative frequencies of errors and error-related responses and the corresponding latencies. Since we had no specific hypotheses concerning these data, they are reported descriptively.

Frequencies. The error rates in the no-ESR and the ESR condition were $22.9 \%$ and $21.9 \%$, respectively. Table 1 shows the relative frequencies of the ESRs, the spontaneous correction responses, and the correct responses in our two conditions. Note that perseverative responses - that is, a correction with the same response-which were very rare $(<0.1 \%)$, were discarded from the analysis. It is remarkable that we observed a relatively high number of spontaneous correction responses in the no-ESR condition $(10.0 \%)$ as well as in the ESR condition (7.6\%). Most important, within the ESR condition, $83.3 \%$ of errors were correctly indicated by an ESR, whereas only $1.6 \%$ of correct responses produced a false alarm.

Response times. Table 1 also depicts the response times of the first response (RT1) as well as those of the correction responses and ESRs (RT2). The latter were determined by the latency between the actual response and the ESR. As expected, the response times of errors were faster than those of correct responses. More- 
Table 1

Relative Frequencies and Latencies of Trials With and Without ESRs and Spontaneous

Corrections Following Errors and Correct Responses From Experiment 2

\begin{tabular}{|c|c|c|c|c|c|c|}
\hline \multirow[b]{2}{*}{ Conditions } & \multicolumn{3}{|c|}{ Correct } & \multicolumn{3}{|c|}{ Error } \\
\hline & Frequency $(\%)$ & RT1 (ms) & RT2 (ms) & Frequency $(\%)$ & RT1 (ms) & $\mathrm{RT} 2(\mathrm{~ms})$ \\
\hline \multicolumn{7}{|c|}{ Condition no-ESR } \\
\hline No-ESR & $99.6(0.7)$ & $350(37)$ & - & $90.0(7.3)$ & $302(36)$ & - \\
\hline Corrected & $0.4(0.6)$ & $337(70)^{\mathrm{a}}$ & $215(220)^{\mathrm{a}}$ & $10.0(7.0)$ & $339(56)$ & $202(108)$ \\
\hline \multicolumn{7}{|l|}{ Condition ESR } \\
\hline No-ESR & $98.2(1.5)$ & $355(35)$ & - & $8.9(9.3)$ & $330(73)^{\mathrm{c}}$ & - \\
\hline Corrected & $0.1(0.4)$ & $324(48)^{\mathrm{b}}$ & $205(134)^{\mathrm{b}}$ & $7.6(4.9)$ & 343 (47) & $163(86)$ \\
\hline ESR & $1.6(1.6)$ & 374 (71) & 438 (107) & $83.3(9.5)$ & 308 (39) & $471(80)$ \\
\hline
\end{tabular}

Note. RT1 and RT2 represent the latencies of the first and the second response (ESR, correction), respectively. The latter was computed as the time elapsing between both responses. Some average latencies are based on a subsample of participants because these trial types did not occur in all participants. The numbers in parentheses are the standard deviations. ESR = error-signaling response.

${ }^{\mathrm{a}} n=9 . \quad{ }^{\mathrm{b}} n=8 .{ }^{\mathrm{c}} n=11$.

over, the response times of spontaneous correction responses were faster than those of the ESRs.

\section{Shift Effects}

In a second step, we examined the effects of errors on subsequent performance. Again, trials following a speed feedback $(1.3 \%)$ and trials following spontaneous error corrections $(3.1 \%)$ were excluded from analyses. In addition, trials following stimulus repetitions $(23.7 \%)$ were also not analyzed. From the remaining data $(72.4 \%)$, we analyzed trials following correct and erroneous responses from the no-ESR condition as well as trials following correct responses without ESR and errors with ESR from the ESR condition. Mean response times of correct responses and error rates were analyzed in a three-way ANOVA with repeated measurement on the factors ESR Mode (ESR, no-ESR), Accuracy on trial $\mathrm{N}-1$ (correct, error), and Task Transition (task repetition, task shift).

Error rates. In the analysis of the error rates, the factor Accuracy on trial $N$-1 was significant, $F(1,13)=13.5, p<.01$. The error rate was reduced following an error $(20.9 \%)$ as compared to following a correct response $(23.7 \%)$. Furthermore, the interaction between Accuracy on trial $N-1$ and Task Transition reached significance, $F(1,13)=12.1, p<.01$. Shift costs were observable after a correct response [repetition: $21.9 \%$, shift: $25.5 \%, t(13)=$ $2.51, p<.05$ ], while shift benefits occurred after an error [repetition: $22.4 \%$, shift: $19.3 \%, t(13)=2.68, p<.01]$. However, there was no significant three-way interaction between all three factors, $F(1,13)<0.01, p=.95$ (cf. Figure 2).

Response times. The same analysis was applied to the response times. Using the same procedure as in Experiment 1, outliers were controlled by excluding $2.5 \%$ of trials. The mean response time was $341 \mathrm{~ms}$. No significant effect was revealed, but there was a trend toward the same pattern as in the error rates. Correct responses were followed by shift costs, and errors were followed by shift benefits in Condition No-ESR [ $\mathrm{N}-1$ correct: repetition: $332 \mathrm{~ms}$, shift: $343 \mathrm{~ms} ; t(13)=2.24, p<.05 ; N-1$ error: repetition: $347 \mathrm{~ms}$, shift: $334 \mathrm{~ms} ; t(13)=1.42, p=.09$ ] as well as in Condition ESR [ $N-1$ correct: repetition: $339 \mathrm{~ms}$, shift: $342 \mathrm{~ms}$; $t(13)=0.72, p=.24 ; N-1$ error: repetition: $348 \mathrm{~ms}$, shift: $343 \mathrm{~ms}$; $t(13)=0.85, p=.21]$. The interaction between Accuracy on trial $N-1$ and Task Transition was marginally significant, $F(1,13)=$ $3.54, p=.08$, while no significant interaction between all three factors was obtained, $F(1,13)=2.33, p=.15$. Again, no (significant) posterror slowing was observable. Response times following errors (343 ms) were comparable to those following correct responses $(339 \mathrm{~ms}), F(1,13)=1.43, p=.25$.

\section{Discussion}

The aim of this experiment was to test whether detected errors cause shift costs or shift benefits on subsequent trials. In half of the blocks, participants were to respond with an ESR in case of an error. In this way, $83.3 \%$ of errors were detected, whereas only $1.6 \%$ of correct responses led to false alarms. Most important, the analysis of shift effects following detected errors revealed that

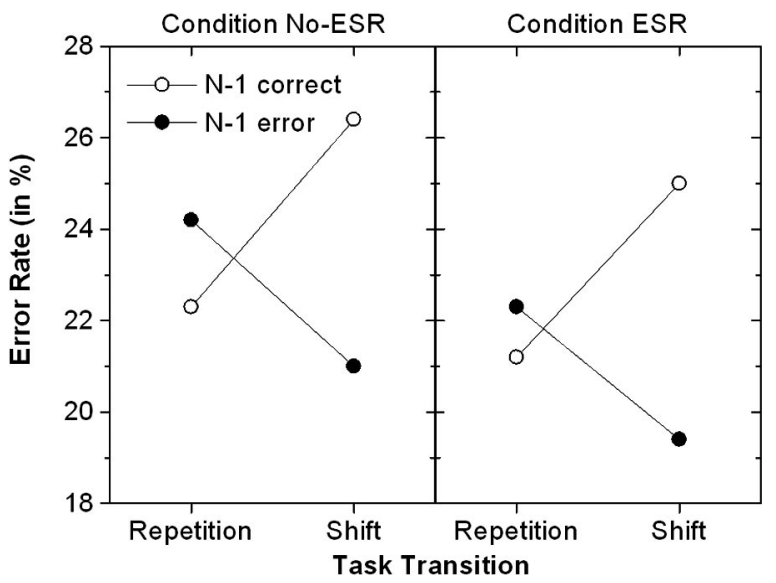

Figure 2. Error rates for task shifts and task repetitions following correct and erroneous responses in the no-ESR and ESR conditions from Experiment 2. Note that only trials following errors with a successful ESR were used in the ESR condition. 
even these errors produced shift benefits on subsequent trials. Moreover, the shift benefits were of the same size as the shift benefits measured in blocks where no ESR was required. Note that this does not imply that detected errors and nondetected errors cause the same shift effect on the subsequent trial. It is reasonable to assume that error detection also occurred in the no-ESR condition. Our results rather show that producing an ESR had no effect on subsequent behavior.

Obviously, shift benefits caused by errors are not due to a lack of posterror processes. Error detection requires that, after producing an erroneous response, processing proceeds until the correct response is determined. Accordingly, in order to detect an error, the correct task has to be activated more strongly than the wrong task after the error occurs. If the shift effects reflect the activation of the tasks at the end of this process, we would expect that shift costs are observable. The fact that shift benefits are obtained shows that this assumption does not hold. Rather, the present results are consistent with a response-based strengthening account of the shift effects. The task that determines an overt response becomes associated with that response, irrespective of whether the activation of the tasks changes after response production. If an error occurs because the wrong task determines the response, then this wrong task becomes associated with the responses. Consequently, a shift to this task in the subsequent trial is beneficial while a task repetition leads to costs.

This account implies that the proactive effect of task-response associations is an all-or-none effect in the sense that either the correct or the wrong task becomes associated with the response. This raises the question of whether this effect is reversible by an immediate error correction. If the outcome of posterror processing is used to correct an error, the correct task should become associated with the response. Consequently, immediate error correction should cause the same shift costs as a correct response.

In order to test this prediction, one could examine those trials where participants corrected their errors spontaneously. Although such spontaneous corrections were observed in Experiments 1 and 2 , their frequency was too low to allow such an analysis. Therefore, we conducted an experiment in which the participants were instructed to correct errors.

\section{Experiment 3}

In this experiment, it should be examined whether corrected errors cause the same shift costs as correct responses (see Figure 3 ). Such a result would support the view that the task that determined the last response also determines the shift effect on the subsequent trial. Shift costs are predicted since error corrections should be caused by the correct task. In order to test this prediction, we replicated Experiment 2, but with a new instruction. Instead of requiring participants to produce an ESR, they were instructed to correct their errors immediately by means of an error-correction response (ECR).

\section{Method}

Twelve participants (10 female, 2 male) between 19 and 26 years of age (mean 22.3) with normal or corrected-to-normal vision participated in the study. Participants were recruited at the University of Konstanz and were paid 5 euros per hour. Design and procedure were the same as in Exper-

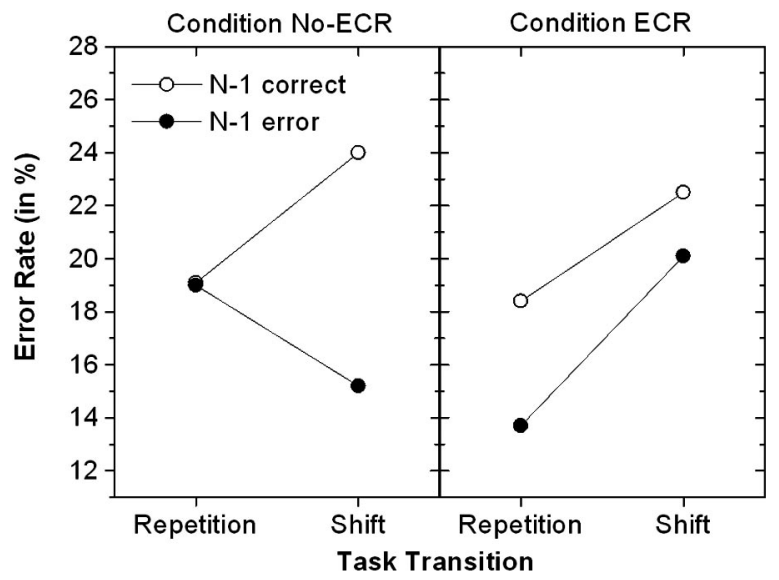

Figure 3. Error rates for task shifts and task repetitions following correct and erroneous responses in the no-ECR and ECR conditions from Experiment 3 . Note that only trials following errors with a successful ECR were used in the ECR condition.

iment 2 with one difference. In half of the blocks, the ECR condition, participants were instructed to correct their errors with an ECR as fast as possible. In the remaining blocks, the no-ECR condition, they were instructed to suppress any spontaneous error corrections.

\section{Results}

\section{Errors and Error-Correction Responses}

Frequencies. Within the no-ECR and ECR conditions, comparable error rates of $20.2 \%$ and $19.6 \%$, respectively, were observed. Table 2 summarizes the relative frequency of trials with a correct ECR and no ECR after erroneous and correct responses for both conditions. Again, the rare cases with perseverative responses $(<0.1 \%)$ were discarded. In the ECR condition, $75.7 \%$ of errors were corrected successfully. It is surprising that $28.3 \%$ of errors were also corrected in the no-ECR condition although participants were explicitly instructed to suppress corrections in this condition. The existence of blocks where an ECR is required seems to enhance the probability of spontaneous corrections in the no-ECR blocks. Only a few correct responses were followed by an ECR in both conditions.

Response times. Again, we report the RT1 and RT2 latencies wherein the latter was computed as the time between the first and the second response. As one would expect (e.g., Fiehler, Ullsperger, \& Von Cramon, 2005), the spontaneous error corrections in the no-ECR condition ( $298 \mathrm{~ms}$ ) were faster than the instructed corrections in the ECR condition (402 ms).

\section{Shift Effects}

For the main analysis, we excluded trials following a speed feedback $(1.4 \%)$ and trials following a stimulus repetition (23.6\%). From the remaining data $(76.3 \%)$, only the following trial types were included: From the no-ECR condition, all trials that were not preceded by an ECR were used. From the ECR condition, only trials following correct responses without ECR and errors with a correct ECR were used. Mean response times of correct responses and error rates were analyzed in three-way ANOVAs with re- 
Table 2

Relative Frequencies of Errors, Correct Responses, Correct ECRs, and Wrong ECRs Within the No-ECR and ECR Conditions From Experiment 3

\begin{tabular}{lrrrrrrrr}
\hline & \multicolumn{3}{c}{ Correct } & & \multicolumn{3}{c}{ Error } \\
\cline { 2 - 3 } \multicolumn{1}{c}{ Conditions } & Frequency & RT1 & RT2 & & Frequency & RT1 & RT2 \\
\hline Condition no-ECR & & & & & & & \\
$\quad$ No-ECR & $99.9(0.3)$ & $340(47)$ & - & & $71.7(19.2)$ & $330(136)$ & - \\
$\quad$ ECR & $0.1(0.3)$ & $332(137)^{\mathrm{a}}$ & $288(259)^{\mathrm{a}}$ & & $28.3(19.2)$ & $313(78)$ & $298(93)$ \\
Condition ECR & & & & & & & \\
$\quad$ No-ECR & $99.4(0.9)$ & $339(49)$ & - & & $24.3(25.4)$ & $353(77)$ & - \\
$\quad$ ECR & $0.6(0.9)$ & $344(83)^{\mathrm{b}}$ & $264(210)^{\mathrm{b}}$ & & $75.7(25.4)$ & $321(81)$ & $402(102)$ \\
\hline
\end{tabular}

Note. RT1 and RT2 represent the latencies of the first and the second response, respectively. The latter was computed as the time elapsing between both responses. Some average latencies are based on a subsample of participants because these trial types did not occur in all participants. The numbers in parentheses are the standard deviations. ECR $=$ error-correction response.

${ }^{\mathrm{a}} n=7 .{ }^{\mathrm{b}} n=10$.

peated measurement on the factors ECR Mode (ECR, no-ECR), Accuracy on trial $\mathrm{N}-1$ (correct, error), and Task Transition (task repetition, task shift).

Error rates. For the error rates, the factor Accuracy on trial $N-1$ was significant, $F(1,11)=17.7, p<.01$. Fewer errors occurred after an error $(17.0 \%)$ than after a correct response $(21.0 \%)$. Moreover, a significant three-way interaction between all factors was revealed, $F(1,11)=9.51, p<.05$. Correct responses always produced shift costs in the subsequent trial [no-ECR: repetition: $19.1 \%$, shift: $24.0 \%, t(11)=2.50, p<.05$; ECR: repetition: $18.4 \%$, shift: $22.5 \%, t(11)=2.50, p<.05]$. However, while errors in the no-ECR condition were followed by shift benefits [repetition: $19.0 \%$, shift: $15.2 \%, t(11)=1.57, p=.07$ ], corrected errors in the ECR condition were followed by shift costs [repetition: $13.7 \%$, shift: $20.1 \%, t(11)=2.78, p<.05$ ]. For a closer examination, we conducted separate two-way ANOVAs for the two ECR conditions with the factors Accuracy on trial $N-1$ and Task Transition. In the no-ECR condition, the interaction between both factors reached significance, $F(1,11)=6.80, p<.05$. In the ECR condition, however, it was not significant, $F(1,11)=0.51$, $p=.49$.

Response times. Outliers were controlled by excluding $2.3 \%$ of trials, using the same procedure as in the preceding experiments. The mean response time was $335 \mathrm{~ms}$. The main effect of Accuracy on trial $\mathrm{N}-1$ was significant, indicating a posterror slowing. Responses were slower following an error $(343 \mathrm{~ms})$ than following a correct response $(326 \mathrm{~ms}), F(1,11)=9.91, p<.01$. In Condition No-ECR, a trend toward the same pattern as in the error rates was obtained with shift costs following correct responses [repetition: $322 \mathrm{~ms}$; shift: $328 \mathrm{~ms} ; t(11)=1.76, p=.053$ ] and shift benefits following errors [repetition: $345 \mathrm{~ms}$, shift: $338 \mathrm{~ms} ; t(11)=1.11$, $p=.15]$. In Condition ECR, almost no shift effects were observed [ $N-1$ correct: repetition: $328 \mathrm{~ms}$, shift: $328 \mathrm{~ms} ; t(11)=0.21, p=$ $.42 ; N$-1 error: repetition: $346 \mathrm{~ms}$, shift: $344 \mathrm{~ms} ; t(11)=0.25, p=$ .40]. The interaction between all three factors was not significant, $F(1,11)=0.90, p=.36$.

Shift effects following spontaneous error corrections in Condition No-ECR and uncorrected errors in Condition ECR. It is surprising that $28.3 \%$ of the errors in Condition No-ECR were spontaneously corrected. Moreover, $24.3 \%$ of the errors in Con- dition ECR remained uncorrected. These relatively high figures allowed us to analyze the shift effects on these trials. Spontaneously corrected errors in Condition No-ECR were followed by nonsignificant shift costs in the error rates [repetition: $16.5 \%$; shift: $18.5 \% ; t(11)=0.54, p=.30]$ as well as in the response times [repetition: $346 \mathrm{~ms}$; shift: $358 \mathrm{~ms} ; t(11)=0.94, p=.19$ ]. For analyzing uncorrected errors in Condition ECR, we could use only a subsample of nine participants because three participants had no uncorrected errors either preceding a task repetition or preceding a task shift. These uncorrected errors produced shift benefits in the error rates [repetition: $27.5 \%$; shift: $18.6 \% ; t(8)=1.90, p<.05$ ] as well as in the response times [repetition: $371 \mathrm{~ms}$; shift: $353 \mathrm{~ms}$; $t(8)=1.03, p=.17]$, whereas only the former reached significance.

\section{Discussion}

The aim of the present experiment was to examine whether corrected and uncorrected errors produce different shift effects on subsequent trials. The results show again that uncorrected errors led to considerable shift benefits. In contrast, corrected errors caused the same shift costs as correct responses. It is interesting that even spontaneous error corrections in blocks where no correction was instructed produced shift costs, while erroneously uncorrected errors caused shift benefits. Obviously, activating the correct task during the posterror period affected subsequent behavior only if this also led to a correction response. This implies that a response is necessary for producing shift effects. Consequently, our response-based strengthening account of the shift effects provides the best account of our data.

Our main argument against the activation-based account is the observation that, although the correct task was activated after the (uncorrected) erroneous response, this did not affect subsequent performance in the same way as correct responses. Accordingly, our reasoning relies on the assumption that error detection implies the activation of the correct task. We make this assumption because, on the one hand, error detection requires that the correct response is activated following an error (e.g., Rabbitt \& Rodgers, 1977; Rabbitt \& Vyas, 1981; Yeung et al., 2004). On the other hand, activating the correct response should be a result of the 
activation of the correct task. Without activating the correct task, it would not be possible to determine the correct response.

That this reasoning is correct is supported by the present results. Error correction is similarly based on the activation of the correct response as error detection. If it would be possible to activate the correct response without activating the correct task, then error correction should have produced not shift costs but the same shift benefits as the detected but not corrected error. That shift costs occurred shows that activating the correct task was necessary for producing an ECR. The same should hold for an ESR.

As announced in the introduction of Experiment 1, we will now examine the response repetition effects of all three experiments in a single section. The corresponding results will add further evidence in favor of our assumptions.

\section{Response-Repetition Effects}

For simplicity, we did not report the response-repetition (RR) effects observed in our individual experiments. However, since these effects play an important role in task-shift studies, they will be reported here in a compact and integrated way. These effects are relevant for the present study for two reasons. First, if we consider other studies, then we find that response repetition usually interacts with task shifting (e.g., Meiran, 2000a; Rogers \& Monsell, 1995; Schuch \& Koch, 2004). Therefore, the contribution of these effects to our shift effects might be relevant. Second, RR effects could indicate which response was activated during posterror processing (Rabbitt \& Rodgers, 1977). Therefore, these effects can be used to test our assumptions regarding the mechanisms underlying error detection.

Usually one observes that, although response repetitions produce benefits on task-repetition trials, they often lead to costs on task-shift trials. Several theories were discussed in the literature to account for this result. Some authors assumed that this pattern is entirely based on the strength (Meiran, 2000a; Rogers \& Monsell, 1995) or the activation (Schuch \& Koch, 2004) of category-response (CR) rules (e.g., less/left). For instance, Meiran (2000a) proposed that task execution leads to a binding between responses and the stimulus categories of the applied task. To account for the RR effects, he further assumed that the actual response is bound more strongly to its category than the alternative response. Because whole CR rules repeat only on task-repetition trials, this can easily explain why RR benefits occur only in this case. Furthermore, he assumed that the association between the actual response and the irrelevant category is weakened at the same time. This would explain why RR costs are observed on task-shift trials.

However, especially to explain RR costs, another mechanism has also been proposed. Rogers and Monsell (1995) considered the possibility that any response is generally inhibited after its production to prevent its accidental reexecution (see also Smith, 1968). Together with the CR-repetition benefits, this could also explain the pattern. On task-shift trials, this inhibition produces RR costs. On task-repetition trials, however, these costs are overcompensated for by the CR repetition. According to Rogers and Monsell, such a two-component account might be better suited because it explains why RR costs and RR benefits are not symmetrical.
Moreover, Hübner and Druey (in press) provided evidence that the magnitude of response inhibition depends on the amount of response activation in the previous trial.

If such a two-component account is valid, then one has to derive separate predictions for the learning component as well as for the inhibition component with respect to the question of how errors affect the interaction between task shifting and response repetition. If response repetition is defined with respect to the actual response in the previous trial, then a response repetition following an error is given when the response required in the current trial is the same as the erroneous one in the previous trial. With respect to the learning component, repeating the CR rule that led to the error should produce an $\mathrm{RR}$ benefit. Assume that the correct $\mathrm{CR}$ association on the previous trial was odd/right. If an error resulted from a task confusion, the rule less/left determined the response. Otherwise, if the error resulted from a response confusion, the rule even/left (or the erroneous rule odd/left) determined the response. As a consequence, following a task confusion, an RR benefit should be observed on task-shift trials because a response repetition implies that less/left is repeated in this case. In contrast, following response confusions, such benefits should be observed, if at all, on task-repetition trials because a response repetition implies that even/left is repeated in this case. Given that the effects of task confusions prevail, the learning component should produce stronger RR benefits on task-shift trials after an error.

With respect to the inhibition component, a different prediction is derived. Originally, it has been assumed that an executed response is inhibited to prevent its accidental reexecution. However, in case of an error, there could be an additional mechanism. Rabbitt and Rodgers (1977) provided evidence that immediately after an error the correct response is also inhibited. They reasoned that this inhibition prevents the system from correcting itself during the extended processing phase. With respect to our effects, this could have the following consequence. It is possible that both responses are inhibited in case of an error, the produced one and the later-activated (and actually correct) one. In other words, the RR costs could disappear after an error because the inhibition of both responses compensates for each other. As a consequence, following an error, RR costs resulting from the inhibition component should either disappear or should be strongly reduced. It is interesting that the same pattern would be expected if the error is corrected. In this case, it is not the later-activated response but the later-produced response that becomes inhibited and counteracts the inhibition of the erroneous response.

If we combine the predictions from the learning component and the inhibition component, the following picture emerges: The inhibition component should determine the general magnitude of RR effects. Following an error, we would expect that generally more RR benefits and less RR costs are observed than following a correct response. In contrast, the learning component should determine to what extent task repetitions and task shifts differ with respect to RR effects. Following an error, we would expect more RR benefits (or less RR costs) on task-shift trials than on taskrepetition trials, whereas the opposite pattern should be observed following a correct response. 


\section{Data Analysis}

RR effects were examined in the present study by extending the main analyses of each experiment by the factor Response Transition (repetition, alternation). A trial was considered an RR trial if the required response was the same as the response given on the preceding trial. Otherwise, it was classified as a responsealternation trial. On trials following corrected errors, response repetition was defined with respect to the correction response. The $\mathrm{RR}$ effects were analyzed separately for each experiment. The data were entered into ANOVAs with repeated measurement on the factors Accuracy on trial $N-1$ (correct, error), Task Transition (repetition, shift), Response Transition (repetition, alternation), and Instruction (ESR, no-ESR in Experiment 2; ECR, no-ECR in Experiment 3). The Congruency factor from Experiment 1 was not included in the analysis. Only significant interactions involving the factor Response Transition are reported.

\section{Results}

The RR effects from all experiments are summarized in Table 3. Generally, our analyses revealed only a few significant effects involving the factor Response Transition. Nevertheless, some consistent trends could be observed across the experiments. We will first summarize these trends descriptively before reporting the results of the ANOVAs.

As can be seen from Table 3, following correct responses, we obtained almost exclusively RR costs in task shifts as well as in task repetitions. The only exception is Experiment 2, in which RR benefits were obtained in task shifts. The magnitude of these RR costs seems not to follow a consistent pattern. They are either the same on task-shift trials and task-repetition trials (Experiment 1, Condition ECR of Experiment 3) or they are subject to a speedaccuracy trade-off (Experiment 2, Condition No-ECR of Experiment 3).

A more consistent pattern is evident following errors. In Experiments 1 and 3, the typical pattern is obtained with RR benefits in task repetitions and RR costs in task shifts, similarly for the error rates as well as for the response times. Only in Experiment 2, RR costs dominate the error rates, which are even stronger on task repetitions relative to task shifts in Condition ESR.
The ANOVAs confirmed these observations only roughly. For Experiment 1, the analysis of the error rates revealed a significant interaction between Accuracy on trial $N-1$ and Response Transition, $F(1,11)=8.50, p<.05$. This reflects the fact that, on average, RR costs of $9.2 \%$ were obtained following correct responses whereas RR benefits of $2.9 \%$ were observed following errors. For the response times, only the interaction between Task Transition and Response Transition reached significance, $F(1$, $11)=5.06, p<.05$, indicating general RR benefits of $4 \mathrm{~ms}$ on task-repetition trials and RR costs of $11 \mathrm{~ms}$ on task-shift trials.

For Experiment 2, there was a significant main effect of Response Transition only on the error rates, $F(1,13)=7.64, p<.05$, which refers to the general RR costs of $5 \%$ observed in this experiment. No effects for the response times were significant.

Finally, for Experiment 3, the main effect of Response Transition, $F(1,11)=5.47, p<.05$, as well as the interaction between Accuracy on trial $N-1$ and Response Transition were significant for the error rates, $F(1,11)=9.48, p<.05$. After a correct response, RR costs of $6.9 \%$ were obtained, whereas weak RR benefits of $0.2 \%$ were observed following errors. For the response times, only the interaction between Task Transition and Response Transition was significant, $F(1,11)=7.13, p<.05$. On average, RR benefits of $6 \mathrm{~ms}$ were observed on task-repetition trials, whereas RR costs of $11 \mathrm{~ms}$ were obtained on task-shift trials.

\section{Discussion}

The RR effects revealed by our analyses partly deviate from the effects typically reported in the literature. Nevertheless, they also partly confirmed our predictions. One prediction concerned the learning component underlying RR effects. This mechanism should determine the relation between RR effects in task repetitions and those in task shifts. Typically, one observes stronger RR benefits (or less RR costs) on task-repetition trials. Following an error, we would have expected the opposite result. Unfortunately, none of these predictions was confirmed. Following correct responses, RR effects did not systematically differ in task repetitions and task shifts. In contrast, following an error, more RR benefits were obtained for task repetitions, whereas RR costs were observed for task shifts. However, these are only trends that did not

Table 3

Response Repetition Benefits From All Conditions of Experiments 1-3

\begin{tabular}{|c|c|c|c|c|c|c|c|c|}
\hline \multirow[b]{3}{*}{ Experiment } & \multicolumn{4}{|c|}{ RT (in ms) } & \multicolumn{4}{|c|}{ Error rate (in \%) } \\
\hline & \multicolumn{2}{|c|}{$\mathrm{N}$-1 correct } & \multicolumn{2}{|c|}{$N-1$ error } & \multicolumn{2}{|c|}{$N$-1 correct } & \multicolumn{2}{|c|}{$N$-1 error } \\
\hline & Task repetition & Task shift & Task repetition & Task shift & Task repetition & Task shift & Task repetition & Task shift \\
\hline Experiment 1 & $-12.3(30.2)$ & $-12.2(24.9)$ & $20.2(91.0)$ & $-9.2(36.0)$ & $-9.2(14.1)$ & $-9.2(12.5)$ & $7.9(20.0)$ & $-2.0(16.8)$ \\
\hline \multicolumn{9}{|l|}{ Experiment 2} \\
\hline No-ESR & $-2.2(37.8)$ & $7.2(24.0)$ & $0.0(41.2)$ & $-8.0(27.7)$ & $-4.9(15.3)$ & $-8.2(10.0)$ & $-2.8(19.8)$ & $-3.0(14.3)$ \\
\hline ESR & $-8.2(43.9)$ & $2.4(33.9)$ & $5.8(33.5)$ & 1.4 (29.6) & $-4.1(17.7)$ & $-7.7(7.8)$ & $-6.8(15.9)$ & $-2.8(17.2)$ \\
\hline \multicolumn{9}{|l|}{ Experiment 3} \\
\hline No-ECR & $-0.3(21.3)$ & $-18.1(24.4)$ & $13.6(39.6)$ & $-2.7(23.4)$ & $-8.6(13.8)$ & $-4.3(12.3)$ & $4.2(11.2)$ & $-3.2(14.0)$ \\
\hline ECR & $-7.8(20.4)$ & $-7.5(29.4)$ & $19.3(44.7)$ & $-18.0(62.4)$ & $-6.9(7.8)$ & $-7.7(12.6)$ & $1.0(10.5)$ & $-1.2(23.4)$ \\
\hline
\end{tabular}

Note. RR benefits are computed by subtracting response times and error rates of response repetitions from those of response alternations. Accordingly, negative values represent RR costs. The numbers in parentheses are the standard deviations. ESR = error-signaling response; ECR $=$ error-correction response. 
reach significance, indicating that our data are rather unstable with respect to these effects. Nevertheless, these results do not suggest that the RR effects substantially contribute to the pattern of shift effects reported in this study. The RR effects strongly vary across and within our experiments, whereas the shift effects are rather consistent.

More interesting were the results regarding the general magnitude of the RR effects following errors and correct responses. Mainly in the error rates, we observed pronounced RR costs on both task-repetition and task-shift trials. However, these costs were present only after correct responses, but not after errors, as indicated by the significant interactions between Accuracy on trial $\mathrm{N}-1$ and Response Transition in Experiments 1 and 3. Figure 4 summarizes the corresponding effects in the error rates for each blocked condition within our experiments. Post hoc tests revealed that, after correct responses, there were always substantial RR costs. After an error, however, there were no such costs (Experiments 1 and 3) or these costs did not reach significance (Experiment 2).

The fact that we observed large RR costs, irrespective of whether the task shifted or repeated, indicates that these effects are due to a general response inhibition mechanism. A first question is why these effects were so strong in our experiments. Indeed, the RR costs are even stronger than the shift effects measured in the error rates. Because our paradigm differs in one important aspect from other task-shift studies, namely by the deadline procedure, it is conceivable that under an extreme speed pressure the risk of an accidental reexecution of the preceding response is very high. This might be due to a very low response threshold. Accordingly, strong inhibition is required to prevent the activation of the response from exceeding the threshold before the stimulus is evaluated in a task-specific way.

The observation that the RR costs disappeared after an error confirms our prediction. Whenever a response was produced, it was inhibited to prevent its reexecution. If the response was an error, then the correct response should be activated during

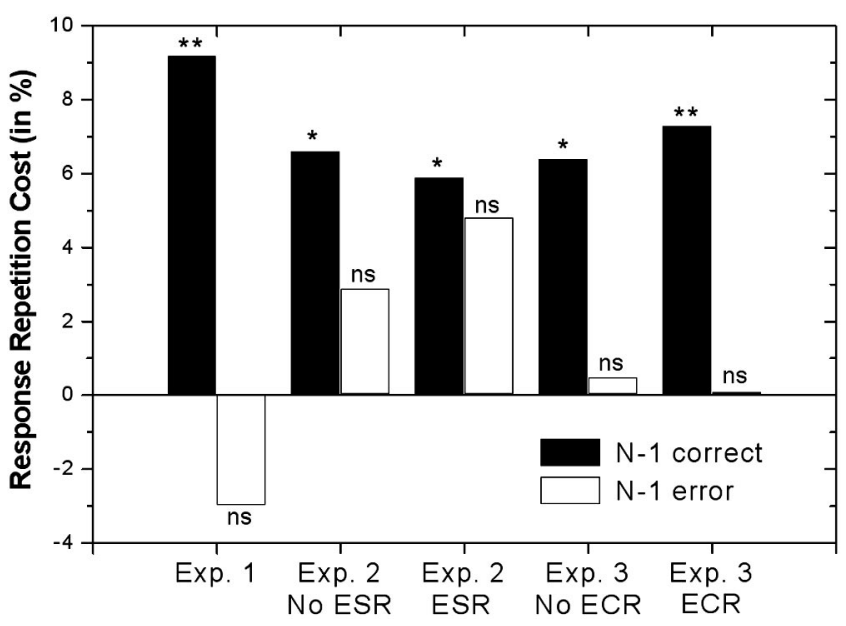

Figure 4. RR cost for the error rates in Experiments 1-3, separately for the blocked conditions following correct and erroneous responses. For each bar, a one-sided $t$ test was computed to evaluate whether the RR effect was reliable ( $n s$ : nonsignificant, $* p<.05, * * p<.01$ ). posterror processing. In this case, there are two possibilities. If the correct response is executed as an ECR, then this response is also inhibited because its accidental reexecution has to be prevented. However, if participants are instructed not to correct their errors, then it can be assumed that, in order to prevent an automatic error correction, the correct response is also inhibited (Rabbitt \& Rodgers, 1977). In either case, both responses are inhibited after a detected error so that neither a response repetition produces benefits nor a response alternation produces costs. Thus, the absence of RR effects following an error provides indirect evidence that error detection implies the activation of the correct response.

Taken together, the analysis of RR effects supports our assumptions concerning error processing. Unfortunately, we were not able to confirm our predictions concerning the learning component underlying the RR benefits. Further research is needed to examine this issue in more detail.

\section{General Discussion}

It is widely assumed that task-shift costs are at least partly due to proactive effects of previous task performance. However, there are diverse ideas about which mechanism causes this effect and, correspondingly, what exactly is reflected by the shift effects. The majority of authors in this field prefer an activation-based account, which states that shift costs represent the relative activation of the tasks resulting from activation and inhibition processes on the previous trial (e.g., Allport et al., 1994; Schuch \& Koch, 2003). Some researchers, however, favor a learning account and assume that the costs reflect the associative strength of the tasks resulting from learning processes on recent trials (e.g., Wylie \& Allport, 2000). Since there is evidence supporting both ideas, we integrated these accounts and proposed a response-based strengthening account. According to this idea, the production of an overt response serves as a cue for strengthening task-response associations, in which the amount of strengthening is proportional to the relative activation of the tasks at the time the response is produced.

The response-based strengthening account predicts that exclusively the relative activation of the tasks before a response should affect subsequent shift effects, whereas any modulation of task activation after the response should not alter these effects. This prediction was tested by examining shift costs following an error. We reasoned that, if the tasks are confused, the wrong task determines the response. After the response, however, processing continues so that finally the correct response becomes activated. Since this processing goes along with the activation of the correct task, a pure activation-based account would predict that after such an erroneous response the same shift costs should be observed as after a correct response. In contrast, from our response-based strengthening account it can be derived that only the state of activation at the time when the erroneous response occurs should affect subsequent performance. Accordingly, since the wrong task determined the response, we should observe shift benefits instead of shift costs after an error.

These predictions were tested in a series of three experiments where we applied the task-shifting paradigm in combination with a deadline procedure. As a result, in each experiment the prediction of the response-based strengthening account was confirmed. The corresponding effects were observed mainly in the error rates. 
In most cases, however, a similar trend was evident in the pattern of response times. Under specific conditions, errors produced shift benefits on the subsequent trial. The results of Experiment 1 verified that these shift benefits were due to task confusions. Only errors for incongruent stimuli produced different shift effects compared to correct responses. This was expected because only for incongruent stimuli a task confusion leads to an error, which implies that the rate of task confusions is higher on trials with errors than on trials with correct responses. A multinomial model applied to our data confirmed that this was the case. However, this model also revealed that even for errors on incongruent stimuli, task confusions were less frequent than correct task applications. To explain why shift benefits could occur in this case, we had to assume that the shift costs resulting from response confusions are generally weaker than the shift benefits resulting from task confusions. This could be a consequence of the fact that response confusions often occur because the dominant task is weakly activated, which implies that less strengthening occurs in this case.

However, the results from Experiment 1 could not allow us to definitely reject the activation-based account because the question remained open whether posterror processing actually took place. Therefore, in Experiment 2 we directly measured error detection by requiring the participants to produce an ESR after each error. This should guarantee that posterror processing took place and activated the correct task. The results show that errors were detected and that this, nevertheless, produced subsequent shift benefits.

Finally, in Experiment 3, we examined whether shift benefits turn into shift costs if errors are immediately corrected by an overt correction response. This was predicted from our response-based strengthening account, given that an additional response initiates further strengthening. As expected, the ECRs led to the same shift costs as correct responses. This demonstrates that the immediate correction of an error is sufficient for also correcting the erroneously learned task associations.

The procedural differences between our experiments also produced different results in some further aspects. For instance, a posterror slowing, that is, an increase in response time after an error, was observed only in Experiments 2 and 3. Furthermore, in these two experiments there were generally smaller error rates and higher response times than in Experiment 1. One reason for this is that the individual deadline intervals in Experiment 1 induced a higher time pressure. This might be due to the fact that this experiment included congruent stimuli, which led to a reduced mean response time in the practice blocks. Since the performance in the practice blocks was used to determine the individual deadline intervals, the deadlines were more difficult to meet in Experiment 1 . The resulting increased time pressure could have led to more errors and a smaller range within which our participants varied their speed-accuracy trade-off. The latter could have prevented strategy changes following errors, which reduced the posterror slowing. However, this should not have influenced our main results. The pattern of shift effects observed in the error rates was almost always present in the response times, too. Thus, it is not possible to explain our results by differences in the posterror slowing in our experiments.

RR effects were also analyzed. For each experiment, the analysis revealed strong repetition costs after a correct response. These costs were interpreted as reflecting response inhibition that should prevent an accidental reexecution (cf. Hübner \& Druey, in press; Rogers \& Monsell, 1995). After an error, however, there were no RR effects irrespective of whether the error was corrected or not. From this pattern of results, we concluded that the correct response was activated even on error trials (see also Rabbitt \& Rodgers, 1977). On these trials, the later-activated correct response was also inhibited in order to prevent automatic correction or (on error correction trials) the accidental reexecution of the correction response. Response repetition effects disappeared because none of the responses had an advantage when both were inhibited. Accordingly, these results provide evidence for the idea that error detection requires the activation of the correct response.

Taken together, our results show that producing an overt response is a necessary precondition for task performance to produce shift effects on the subsequent trial. Applying a task affected subsequent performance only if this task led to an overt response (i.e., a correct response, an error, or a correction response). In contrast, applying a task in order to detect an error was not sufficient to produce a proactive effect of this task. The crucial role of the overt response suggests that shift effects are produced by a process that is linked to response production. This contradicts the widely held assumption that shift costs directly reflect the relative activation of the tasks. However, our results are in line with a response-based strengthening account.

In the following paragraphs, we will consider some assumptions which are crucial for our conclusions. Then, we will discuss further empirical evidence from the literature in the light of our account. Finally, we will derive some implications of our results for the question of how errors affect subsequent performance in general.

\section{What Causes Task Confusions?}

One of our assumptions concerns the source of task confusions that cause the shift benefits on subsequent trials. We assumed that they result from the failure of a selection process that normally inhibits the irrelevant task when this task was also activated by the stimulus (see Mayr, 2001; Steinhauser \& Hübner, in press-b). Accordingly, we suggested that the source of the task confusions is located in poststimulus processes.

Alternatively, however, one could assume that task confusions result from the failure of task preparation that occurs before the stimulus appears. For instance, the tasks could be confused because the cue was misinterpreted. In this case, participants would deliberately perform the wrong task. As a consequence, a task shift in the subsequent trial becomes a task repetition and vice versa. This could perfectly explain our main results. However, such an interpretation contradicts some of our data. If the cues had been misinterpreted, the resulting errors would not have been detectable since our participants would have been convinced that they had performed the correct task. In contrast to this scenario, we showed that errors causing shift benefits on the subsequent trial were reliably detected. This indicates that our participants were aware of the required task, which is at odds with the assumption that they misinterpreted the cue.

Another alternative source for task confusions could be that, at least on some trials, participants did not prepare sufficiently for the indicated task in spite of the long cue-stimulus interval (CSI), as proposed by De Jong (2000). Such an explanation receives support 
from Altmann (2004), who found that long CSIs were used for efficient preparation only when participants were also confronted with short CSIs. It seems that participants are not quite motivated when the CSI is constant. However, even if the observed task confusions were a consequence of a suboptimal preparation, this would not contradict our interpretation of the shift benefits following these errors. For our conclusions, it is relatively unimportant whether task confusions occur because a poststimulus inhibition process failed or because the inhibition during the preparation phase went wrong. In both cases, the irrelevant task produced an error because its activation was stronger than that of the relevant task.

\section{Error Detection and Task-Set Activation}

Our conclusions regarding the source of shift costs are strongly based on the assumption that error detection implies that the correct task is activated even after task confusion. This assumption was derived from two premises. First, errors are detected because the correct response is activated immediately after the error. Second, activating the correct response is possible only if the correct task is activated first. The first premise is suggested by the extended processing hypothesis (Rabbitt \& Vyas, 1981) and receives support from our analyses of RR effects. The second premise, however, was logically derived from the first one. It is hardly conceivable that a response-selection mechanism can reliably determine the correct response when the wrong task dominates processing. Thus, the validity of our conclusions mainly depends on whether the first premise is valid. The question arises whether there is a way of detecting errors without activating the correct response.

At first sight, it seems that such a way is given by the theory of conflict-based error detection introduced by Yeung et al. (2004). This theory is based on the idea that response activation is continuously monitored by a mechanism located in the anterior cingulate cortex (Botvinick, Braver, Barch, Carter, \& Cohen, 2001). This mechanism generates a conflict signal that is high whenever two contradictory responses are activated concurrently. In simulations with a neural network model, Yeung and colleagues could show that such a conflict signal is sufficient to detect errors in an Eriksen flanker paradigm (Eriksen \& Eriksen, 1974) in which participants have to respond to a target letter while ignoring several distractor letters. Such a conflict-based error detection is possible because response conflicts are rather strong immediately after an error. In their model, an error is detected whenever the accumulated posterror conflict exceeds a threshold.

However, a closer inspection of this model shows that it does not contradict our premises. Since the posterror response conflict is caused by the activation of the correct response, the theory is clearly in line with the extended processing hypothesis. Moreover, the model even supports our second suggestion. A portion of errors in their simulations occur because a response is produced before the attentional set is sufficiently established. Due to noise, the distractor stimuli can elicit a response before the target letter is fully activated by an attentional bias. Indeed, this roughly corresponds to a task-confusion error in our framework. However, after such an error, the correct attentional set finally prevails and allows for the activation of the correct response. Thus, the conflict-based error-detection account of Yeung et al. (2004) perfectly supports our premises regarding the conditions under which errors are detected.

\section{Response-Based Strengthening}

Our approach to explain the source of the residual shift costs has two components. First, we assumed that the costs reflect a proactive effect of task learning, and, second, we suggested that this learning occurs only in the context of an overt response. The experiments reported in this study addressed only the second component. However, the suggestion that residual shift costs are due to associative learning receives support from other studies. For distinguishing a learning effect from an activationbased effect, it is helpful to consider its temporal stability. Whereas learning effects should be rather stable, activationbased effects might decay over time. For the shift costs, this has been tested in studies that manipulated the interval between the response and the following cue (e.g., Meiran, Chorev, \& Sapir, 2000). It turned out that the shift costs decreased with an increasing interval. However, even an interval with a duration of three seconds did not eliminate the costs (Meiran et al., 2000). Moreover, whether an effect of the response-cue interval on the shift costs is observed seems to depend on whether this interval is manipulated within subject or between subjects, which contradicts the idea that the source of this effect is the decay of activation (Altmann, 2005). These results suggest that the proactive effect underlying the residual shift costs is rather stable and does not decay over time.

Furthermore, there is some evidence that the shift costs depend on the frequency of which the tasks occurred on previous trials, which is consistent with a learning account. For instance, it has been shown that, at least for some task-shifting procedures, the shift costs dissipate gradually across several task repetitions after a task shift (e.g., Hübner, Futterer, \& Steinhauser, 2001; Monsell, Sumner, \& Waters, 2003). This suggests that with each repetitive execution of a given task, the associative weights of this task become stronger. At the same time, the shift cost on a given trial depends on how many task repetitions preceded this trial (Ruthruff et al., 2001). A shift toward Task A produces higher shift costs if this was preceded by many repetitions of another Task B than if this was preceded by only a few repetitions of Task B (but see also Mayr \& Keele, 2000). This could reflect the fact that the associative strength of Task A determines the amount of shift costs when shifting toward Task B. Finally, the overall frequency of the tasks performed is also crucial for the amount of shift costs (Wylie \& Allport, 2000). These effects seem to depend on the specific paradigm and the specific tasks that are used. Future research should reveal the boundary conditions under which cumulative effects of task performance can be observed.

Another question which was not directly addressed by our experiments concerns the type of associations that were strengthened. We adopted the idea from Meiran (2000a) that the residual shift effects are based on associations between taskrelevant categories (e.g., odd, even) and responses. However, taking other results into account, it is plausible to assume that it is not the response itself that is part of these associations, but rather an abstract response category like left. This is suggested by studies showing that effects of response-category repetition 
as well as effects of congruency emerge even when the tasks are assigned to different hands (Hübner \& Druey, in press; Schuch $\&$ Koch, 2004). Basically, the idea that response-related associations are involved was motivated by the observation that residual shift costs are observed only when both tasks make use of the same responses or response categories (Meiran, 2000b) and that the task shift effects interact with response repetition effects (e.g., Meiran, 2000a). However, since the latter result was not replicated by our data, we cannot exclude the potentiality that the shift effects observed here are due to the strengthening of other types of associations. For instance, it is possible that our effects are due to associations mediating attention to stimulus dimensions (the S-set in the model of Meiran, 2000a) or even associations between the cues and task-related representations $^{5}$ (for the role of the cue in shift costs, see Koch, 2003; Logan \& Bundesen, 2003; Mayr \& Kliegl, 2003).

A further question is how strengthening is triggered. For our response-based strengthening account, we assume that an overt response provides a cue for strengthening the task. From a theoretical perspective, this assumption could be motivated by the idea that an overt response is a valid indication that the task that triggered the response was the most activated. It makes sense that at this time a reinforcement signal is given that triggers the strengthening of associations. However, we do not think that the response itself is such a signal. In our data, we found that not every type of response led to strengthening. If the activation of the correct response during posterror processing was utilized for producing an ESR, this did not lead to strengthening. From this result, one can conclude that only a response that is linked to the task set is able to initiate learning of the corresponding task. This holds for an ECR but not for an ESR.

Another important question pertains to the nature of the learning mechanism. For explaining the shift effects, learning should have at least two properties. First, it is unsupervised; that is, error detection does not prevent the associations of the wrong task from being strengthened. This assumption is necessary to explain why shift benefits following errors are observed. Second, learning is gradual; that is, the amount of strengthening is proportional to the activation of the tasks. In this way, one can explain why the magnitude of the shift costs depends on, for instance, how much inhibition was applied in the preceding trial (e.g., Allport et al., 1994). A learning algorithm that fulfills these two properties is correlation-based Hebbian learning in neural networks, in which the associative strength between two units is changed proportionally to the product of their activation. Indeed, Gilbert and Shallice (2002) used such an algorithm to model item-specific effects in task shifting.

Finally, the present study also contributes to an understanding of the general effects of errors on subsequent behavior. The idea that erroneous and correct behavior is strengthened in a comparable way is rather old (for a brief review, see Pashler, Zarow, \& Triplett, 2003). For instance, the notion that nondeclarative memory is based on unsupervised learning has been proposed to account for specific learning deficits in patients with medialtemporal lesions (McClelland, 2001). However, Pashler et al. recently concluded that, so far, there is no direct experimental evidence for the learning of errors. If our interpretation of the present results is valid, our data provide evidence that there is learning of errors and that this occurs even when errors are detected.

\section{Conclusion}

In three experiments, we showed that erroneous task application resulting from task confusions causes shift benefits instead of shift costs on subsequent trials. This also held for detected errors, whereas ECRs produced the same shift costs as correct responses. These results imply that the proactive effects that are reflected in these shift costs depend on the production of a response. The activation of tasks alone is not sufficient. This is consistent with theories that explain proactive effects of task performance with a learning or binding process that strengthens the associations between tasks and responses.

\footnotetext{
${ }^{5}$ Note that, although the cues might be involved in associative learning, our results cannot be explained in terms of the priming of cue encoding, as proposed by Logan and Bundesen (2003) or Schneider and Logan (2005). This idea was meant to account for the shift costs at short CSIs, which were originally explained in terms of a reconfiguration process. Moreover, to explain shift benefits following task confusions in terms of a cue encoding effect, one would have to assume that task confusions are due to cue encoding failures (i.e., the encoding of the wrong cue). In this case, however, the participants should not be aware of having committed an error, as discussed earlier.
}

\section{References}

Allport, A., Styles, E. A., \& Hsieh, S. (1994). Shifting intentional set: Exploring the dynamic control of tasks. In C. Umiltà \& M. Moscovitch (Eds.), Attention and performance XV: Conscious and nonconscious information processing (pp. 421-452). Cambridge, MA: MIT Press.

Altmann, E. M. (2004). Advance preparation in task switching: What work is being done? Psychological Science, 15, 616-622.

Altmann, E. M. (2005). Repetition priming in task switching: Do the benefits dissipate? Psychonomic Bulletin \& Review, 12, 535-540.

Batchelder, W. H., \& Riefer, D. M. (1990). Multinomial processing models of source monitoring. Psychological Review, 97, 548-564.

Botvinick, M. M., Braver, T. S., Barch, D. M., Carter, C. S., \& Cohen, J. D. (2001). Conflict monitoring and cognitive control. Psychological Review, 108, 624-652.

De Jong, R. (2000). An intention-activation account of residual switch costs. In S. Monsell \& J. Driver (Eds.), Control of cognitive processes: Attention and performance XVIII (pp. 357-376). Cambridge, MA: MIT Press.

Eriksen, B. A., \& Eriksen, C. W. (1974). Effects of noise letters upon the identification of a target letter in a nonsearch task. Perception \& Psychophysics, 16, 143-149.

Fairweather, H. (1978). Choice reaction times in children: Error and post-error responses, and the repetition effect. Journal of Experimental Child Psychology, 26, 407-418.

Fiehler, K., Ullsperger, M., \& Von Cramon, D. Y. (2005). Electrophysiological correlates of error correction. Psychophysiology, 42, 72-82.

Gilbert, S. J., \& Shallice, T. (2002). Task switching: A PDP model. Cognitive Psychology, 44, 297-337.

Goschke, T. (2000). Intentional reconfiguration and involuntary persistence in task set switching. In S. Monsell \& J. Driver (Eds.), Control of cognitive processes: Attention and performance XVIII. Cambridge, MA: MIT Press.

Hoffmann, J., Kiesel, A., \& Sebald, A. (2003). Task switches under 
Go/NoGo conditions and the decomposition of switch costs. European Journal of Cognitive Psychology, 15, 101-128.

Hübner, R., \& Druey, M. (in press). Response execution, selection, or activation: What is sufficient for response-related repetition effects under task shifting? Psychological Research.

Hübner, R., Futterer, T., \& Steinhauser, M. (2001). On attentional control as a source of residual shift costs: Evidence from two-component task shifts. Journal of Experimental Psychology: Learning, Memory, and Cognition, 27, 640-653.

Hübner, M., Kluwe, R. H., Luna-Rodriguez, A., \& Peters, A. (2004). Response selection difficulty and asymmetrical costs of switching between tasks and stimuli: No evidence for an exogenous component of task-set reconfiguration. Journal of Experimental Psychology: Human Perception and Performance, 30, 1043-1063.

Kleinsorge, T., \& Gajewski, P. D. (2004). Preparation for a forthcoming task is sufficient to produce subsequent shift costs. Psychonomic Bulletin \& Review, 11, 302-306

Koch, I. (2003). The role of external cues for endogenous advance reconfiguration in task switching. Psychonomic Bulletin \& Review, 10, $488-$ 492.

Laming, D. (1979a). Autocorrelation of choice-reaction times. Acta Psychologica, 43, 381-412.

Laming, D. (1979b). Choice reaction performance following an error. Acta Psychologica, 43, 199-224.

Logan, G. D., \& Bundesen, C. (2003). Clever homunculus: Is there an endogenous act of control in the explicit task-cuing procedure? Journal of Experimental Psychology: Human Perception and Performance, 29, $575-599$.

Mayr, U. (2001). Age differences in the selection of mental sets: The role of inhibition, stimulus ambiguity, and response-set overlap. Psychology and Aging, 16, 96-109.

Mayr, U., \& Keele, S. W. (2000). Changing internal constraints on action: The role of backward inhibition. Journal of Experimental Psychology: General, 129, 4-26.

Mayr, U., \& Kliegl, R. (2003). Differential effects of cue changes and task changes on task-set selection costs. Journal of Experimental Psychology: Learning, Memory, and Cognition, 29, 362-372.

McClelland, J. L. (2001). Failures to learn and their remediation: A Hebbian account. In J. L. McClelland \& R. S. Siegler (Eds.), Mechanisms of cognitive development: Behavioral and neural perspectives ( $\mathrm{pp}$. 97-121). Mahwah, NJ: Erlbaum.

Megaw, E. D. (1972). Directional errors and their correction in a discrete tracking task. Ergonomics, 15, 633-643.

Meiran, N. (1996). Reconfiguration of processing mode prior to task performance. Journal of Experimental Psychology: Learning, Memory, and Cognition, 22, 1423-1442.

Meiran, N. (2000a). Modeling cognitive control in task-switching. Psychological Research, 63, 234-249.

Meiran, N. (2000b). Reconfiguration of stimulus task sets and response task sets during task switching. In S. Monsell \& J. Driver (Eds.), Control of cognitive processes: Attention and performance XVIII (pp. 377-400). Cambridge, MA: MIT Press.

Meiran, N., Chorev, Z., \& Sapir, A. (2000). Component processes in task switching. Cognitive Psychology, 41, 211-253.

Meiran, N., \& Gotler, A. (2001). Modelling cognitive control in task switching and ageing. European Journal of Cognitive Psychology, 13, 165-186.

Monsell, S., Sumner, P., \& Waters, H. (2003). Task-set reconfiguration with predictable and unpredictable task switches. Memory \& Cognition, 31, 327-342.

Pashler, H., Zarow, G., \& Triplett, B. (2003). Is temporal spacing of tests helpful even when it inflates error rates? Journal of Experimental Psychology: Learning, Memory, and Cognition, 29, 1051-1057.
Rabbitt, P. (1967). Time to detect errors as a function of factors affecting choice-response time. Acta Psychologica, 27, 131-142.

Rabbitt, P. (1968). Three kinds of error-signalling responses in a serial choice task. Quarterly Journal of Experimental Psychology, 20, 179188.

Rabbitt, P. (1990). Age, IQ and awareness, and recall of errors. Ergonomics, 33, 1291-1305.

Rabbitt, P. (2002). Consciousness is slower than you think. Quarterly Journal of Experimental Psychology: Human Experimental Psychology, 55, 1081-1092.

Rabbitt, P., \& Rodgers, B. (1977). What does a man do after he makes an error? An analysis of response programming. Quarterly Journal of Experimental Psychology, 29, 727-743.

Rabbitt, P., \& Vyas, S. (1981). Processing a display even after you make a response to it: How perceptual errors can be corrected. Quarterly Journal of Experimental Psychology: Human Experimental Psychology, 33, 223-239.

Ridderinkhof, K. R. (2002). Micro- and macro-adjustments of task set: Activation and suppression in conflict tasks. Psychological Research Psychologische Forschung, 66, 312-323.

Rogers, R. D., \& Monsell, S. (1995). Costs of a predictable switch between simple cognitive tasks. Journal of Experimental Psychology: General, 124, 207-231.

Ruthruff, E., Remington, R. W., \& Johnston, J. C. (2001). Switching between simple cognitive tasks: The interaction of top-down and bottom-up factors. Journal of Experimental Psychology: Human Perception and Performance, 27, 1404-1419.

Scheffers, M. K., \& Coles, M. G. (2000). Performance monitoring in a confusing world: Error-related brain activity, judgments of response accuracy, and types of errors. Journal of Experimental Psychology: Human Perception and Performance, 26, 141-151.

Schneider, D. W., \& Logan, G. D. (2005). Modeling task switching without switching tasks: A short-term priming account of explicitly cued performance. Journal of Experimental Psychology: General, 134, 343-367.

Schuch, S., \& Koch, I. (2003). The role of response selection for inhibition of task sets in task shifting. Journal of Experimental Psychology: Human Perception and Performance, 29, 92-105.

Schuch, S., \& Koch, I. (2004). The costs of changing the representation of action: Response repetition and response-response compatibility in dual tasks. Journal of Experimental Psychology: Human Perception and Performance, 30, 566-582.

Smith, M. C. (1968). Repetition effect and short-term memory. Journal of Experimental Psychology, 77, 435-439.

Sohn, M.-H., \& Anderson, J. R. (2001). Task preparation and task repetition: Two-component model of task switching. Journal of Experimental Psychology: General, 130, 764-778.

Steinhauser, M., \& Hübner, R. (in press-a). Automatic activation of taskrelated representations in task-shifting. Memory \& Cognition.

Steinhauser, M., \& Hübner, R. (in press-b). Mixing costs in task-shifting reflect sequential processing stages in a multi-component task. Memory \& Cognition.

Waszak, F., Hommel, B., \& Allport, A. (2003). Task-switching and longterm priming: Role of episodic stimulus-task bindings in task-shift costs. Cognitive Psychology, 46, 361-413.

Winer, B. J., Brown, D. R., \& Michels, K. M. (1991). Statistical principles in experimental design (3rd ed.). Boston: McGraw-Hill.

Wylie, G., \& Allport, A. (2000). Task switching and the measurement of "switch costs." Psychological Research, 63, 212-233.

Yeung, N., Cohen, J. D., \& Botvinick, M. M. (2004). The neural basis of error detection: Conflict monitoring and the error-related negativity. Psychological Review, 111, 939-959. 


\section{Appendix}

By means of multinomial models, we can estimate the frequency of unobservable events from the frequencies of observable events (Batchelder \& Riefer, 1990). In our case, we can merely observe whether a participant produced the required response $\mathrm{C}$ or an error $\mathrm{E}$ and whether the stimulus was congruent or incongruent. However, we cannot observe whether an error resulted from a task confusion or from a response confusion. To determine the frequencies of these events, we can construct a tree diagram (see Figure A1) that describes the possible events leading to a specific response. We assume that participants apply either the correct task T or the alternative task $\mathrm{T}^{\prime}$. Then they select either the correct response $\mathrm{R}$ or the wrong response $\mathrm{R}^{\prime}$ for the task applied.

The crucial point is that the corresponding pathways for congruent and incongruent stimuli in Figure A1 can lead to different outcomes. This can be demonstrated regarding the example in Figure A1. For both stimuli 2 and 3, applying the correct task $\mathrm{T}$ (magnitude) leads to a correct response $\mathrm{C}$ if $\mathrm{R}$ (less/left) is selected, whereas it leads to an error if $\mathrm{R}^{\prime}$ (greater/right) is selected. If, however, the wrong task $\mathrm{T}^{\prime}$ (parity) is applied, the results differ. For the congruent stimulus, R (even/left) implies a correct response, while R' (odd/right) corresponds to an error. In contrast, for the incongruent stimulus, selecting the correct response $\mathrm{R}$ for the wrong task (odd/right) leads to an error, whereas selecting the wrong response $\mathrm{R}^{\prime}$ for the wrong task (even/left) causes a correct response.

Given this description, we can now calculate the probabilities of the different events. A first step toward this goal is to compute the probability for each single pathway in our tree. Consider the case that the correct task $\mathrm{T}$ is applied with the probability $p(\mathrm{~T})$, and the corresponding correct response $\mathrm{R}$ is selected with the probability $p(\mathrm{R})$. Given that $p(\mathrm{~T})$ and $p(\mathrm{R})$ are stochastically independent, their joint probability is $p(\mathrm{~T}) p(\mathrm{R})$. In this way, we can calculate the probability of each pathway in our tree diagram.

The total probability of an error $p(\mathrm{E})$ is simply the sum of the probabilities for those pathways that lead to an error. In case of a congruent stimulus, errors can occur either because the correct task T was applied but the wrong response $\mathrm{R}^{\prime}$ was selected, or because the wrong task $\mathrm{T}^{\prime}$ was applied and the wrong response $\mathrm{R}^{\prime}$ was selected. Because we have binary events, we can also write the probabilities of task confusions $p\left(\mathrm{~T}^{\prime}\right)$ and of response confusions $p\left(\mathrm{R}^{\prime}\right)$ as 1- $p(\mathrm{~T})$ and1- $p(\mathrm{R})$, respectively. In this case, the error probability $p_{c o n}$ for congruent stimuli is

$$
p_{c o n}(\mathrm{E})=p(\mathrm{~T})(1-p(\mathrm{R}))+(1-p(\mathrm{~T}))(1-p(\mathrm{R})) .
$$

Solving for $p(\mathrm{R})$ and $p\left(\mathrm{R}^{\prime}\right)$ results in

$$
p(\mathrm{R})=1-p_{c o n}(\mathrm{E}) \text { and } p\left(\mathrm{R}^{\prime}\right)=p_{c o n}(\mathrm{E}) .
$$

As can be seen, the error rate for congruent stimuli is an estimator for the rate of response confusions $p\left(\mathbf{R}^{\prime}\right)$. This simply reflects the fact that, for congruent stimuli, only a response confusion leads to an error irrespective of whether the correct task or the wrong task was applied.

Now, we can consider the performance for incongruent stimuli to compute the probability of task confusions. We have

$$
p_{\text {inc }}(\mathrm{E})=p(\mathrm{~T})(1-p(\mathrm{R}))+(1-p(\mathrm{~T})) p(\mathrm{R}) .
$$

Solving for $p(\mathrm{~T})$ results in

$$
p(\mathrm{~T})=\frac{p_{\text {inc }}(\mathrm{E})-p(\mathrm{R})}{1-2 p(\mathrm{R})}=\frac{p_{\text {inc }}(\mathrm{E})+p_{\text {con }}(\mathrm{E})-1}{2 p_{\text {con }}(\mathrm{E})-1} .
$$

Substituting the terms by the corresponding empirical error rates from our Experiment 1, we obtain for $p(\mathrm{~T})$ and $p(\mathrm{R})$ the values 0.91 and 0.78 , respectively. With these values, we can now determine the probabilities of task confusions for congruent and incongruent stimuli on correct and on error trials:

$$
\begin{gathered}
p_{c o n}\left(T^{\prime} \mid E\right)=\frac{1}{p_{c o n}(E)}(1-p(T))(1-p(R))=1-p(T) \\
p_{c o n}\left(T^{\prime} \mid C\right)=\frac{1}{1-p_{c o n}(E)}(1-p(T)) p(R)=1-p(T)
\end{gathered}
$$

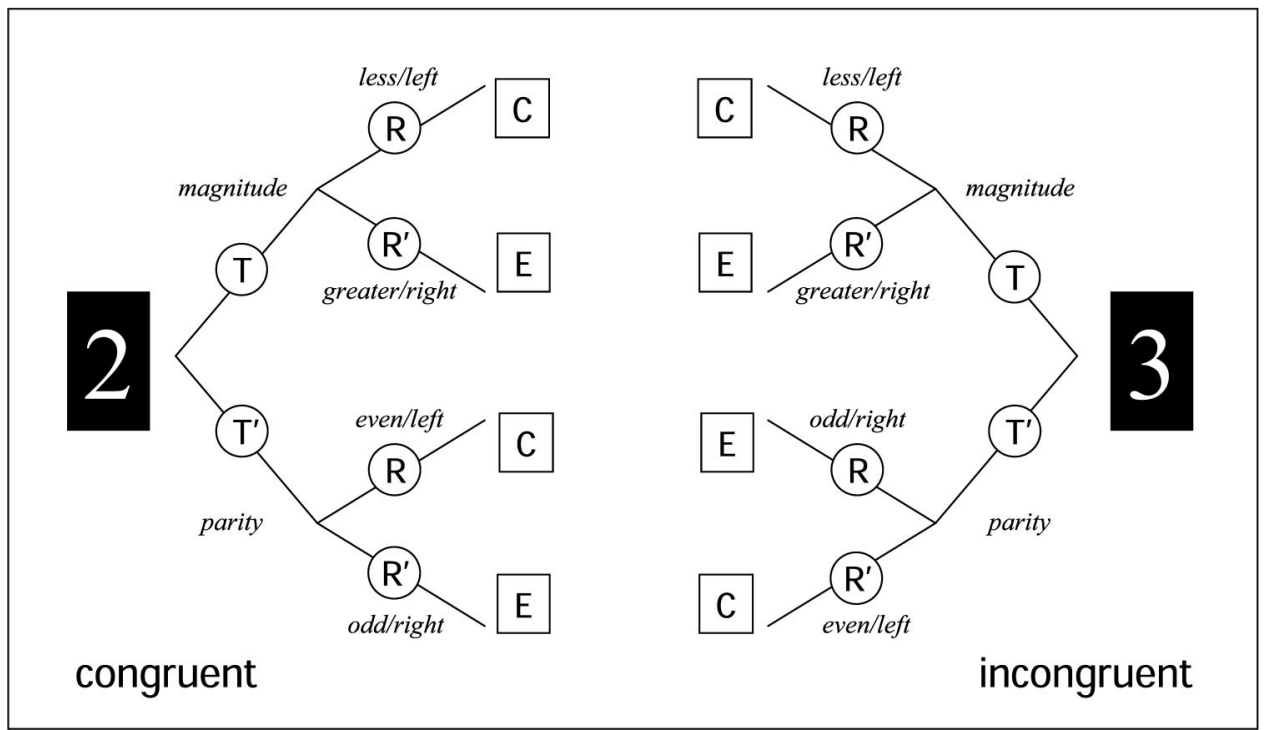

Figure A1. Illustration of a multinomial model of the processes involved in our tasks, for a congruent stimulus (left) and an incongruent stimulus (right). Squares denote observable events whereas circles denote unobservable events. Abbreviations: $\mathrm{T} / \mathrm{T}^{\prime}$ : correct and incorrect task, respectively; $\mathrm{R} / \mathrm{R}^{\prime}$ : correct and incorrect response, respectively, with respect to the task applied; C: correct trial; E: error trial. 
534

$$
\begin{gathered}
p_{\text {inc }}\left(T^{\prime} \mid E\right)=\frac{1}{p_{\text {inc }}(E)}(1-p(T)) p(R) \\
p_{\text {inc }}\left(T^{\prime} \mid C\right)=\frac{1}{1-p_{\text {inc }}(E)}(1-p(T))(1-p(R))
\end{gathered}
$$

occurred with probability 0.090 on correct trials, which is identical to that on error trials. In contrast, for incongruent stimuli, the probability of a task confusion on correct trials was 0.027 , whereas that on error trials was 0.263 .

With these formulas we obtained the following estimates for task confusions in our first experiment: For congruent stimuli, a task confusion 